International Journal of Modern Physics D Vol. 25, No. 14 (2016) 1699001 (14 pages)

(C) World Scientific Publishing Company

DOI: $10.1142 / \mathrm{S} 0218271816990017$

\title{
Cumulative author index
}

Abdolmaleki, A. \& Najafi, T., Generalized second law of thermodynamics on the apparent horizon in modified Gauss-Bonnet gravity

Ablu Meitei, I., see Ibungochouba Singh

Abri, A., see Moradpour

Acuña, J. T. \& Esguerra, J. P., Dynamics of a planar thin shell at a Taub-FRW junction

Adler, S. L., A framedependent gravitational effective action mimics a cosmological constant, but modifies the black hole horizon

Aftabi, S., see Salehi

Ahmed, W., Ishaque, O. \& Rehman, M. U., Quantum smearing in hybrid inflation with chaotic potentials

Akhshabi, S., see Behboodi

Alesci, E. \& Cianfrani, F., Quantum reduced loop gravity and the foundation of loop quantum cosmology

Alexander, S., Inflationary birefringence and baryogenesis

Alhamzawi, A. \& Alhamzawi, R., Gravitational lensing by $f(R, T)$ gravity

Alhamzawi, R., see Alhamzawi

Ali, A. F. \& Das, S., Stringent theoretical and experimental bounds on graviton mass

Allahyari, A., Firouzjaee, J. T. \& Mansouri, R.,

Gravitational collapse in the AdS background and the black hole formation

Alpin, T. Yu. \& Balakin,

25 (2016) 1650040

25 (2016) 1650061

25 (2016) 1650014

25 (2016) 1650001

25 (2016) 1643001

25 (2016) 1650042

25 (2016) 1650035

25 (2016) 1650087

25 (2016) 1642005

25 (2016) 1640013

25 (2016) 1650020

25 (2016) 1650020

25 (2016) 1644001
A. B., The EinsteinMaxwell-aether-axion theory: Dynamo-optical anomaly in the electromagnetic response

Amani, A. R., The bouncing cosmology with $F(R)$ gravity and its reconstructing

Amati, L., see Frontera

Amelino-Camelia, G., Gubitosi, G. \& Palmisano, G., Pathways to relativistic curved momentum spaces: de Sitter case study

Anderson, E., Six new mechanics corresponding to further shape theories

André, R., see Kremer

Arik, M. \& Senikoglu, Y., Cosmological isotropic matter-energy generalizations of Schwarzschild and Kerr metrics

Arkhipova, N. A. \& Pilipenko, S. V., Prospects for studying the dark energy at $z>2$ with galaxy surveys

Ashtekar, A., Symmetry reduced loop quantum gravity: A bird's eye view

Ault, K. L., see Varieschi

Ayón-Beato, E., Canfora, F. \& Zanelli, J., Selfgravitating skyrmions

Babar, G. Z., Jamil, M. \& Lim, Y.-K., Dynamics of a charged particle around a weakly magnetized naked singularity

Bakke, K., see Belich
25 (2016) 1650005

25 (2016) 1650048

25 (2016) 1650071

25 (2016) 1630014

25 (2016) 1650027

25 (2016) 1650044 25 (2016) 1650012

25 (2016) 1650088

25 (2016) 1650109

25 (2016) 1642010 25 (2016) 1650064

25 (2016) 1641009

25 (2016) 1650024

25 (2016) 1641003 
Balakin, A. B., see Alpin

Balart, L. \& Peña, F., Regular charged black holes, quasilocal energy and energy conditions

Bañados, M. \& Reyes, I., A short review on Noether's theorems, gauge symmetries and boundary terms

Banerjee, S., Bera, S. \& Singh, T. P., Quantum nonlocality and the end of classical spacetime

Barrau, A. \& Bolliet, B., Some conceptual issues in loop quantum cosmology

Basilakos, S., see Paliathanasis

Battistelli, E. S., Burigana, C., de Bernardis, P., Kirillov, A. A., Neto, G. B. L., Masi, S., NorgaardNielsen, H. U., Ostermann, P., Roman, M., Rosati, P. \& Rossetti, M., Galaxy clusters as probes for cosmology and dark matter

Battistelli, E. S., see Burigana

Beetle, C., Engle, J. S., Hogan, M. E. \& Mendonça, P., Diffeomorphism invariant cosmological symmetry in full quantum gravity

Behboodi, A., Akhshabi, S. \& Nozari, K., Scalar perturbation potentials in a homogeneous and isotropic Weitzenböck geometry

Belich, H. \& Bakke, K., Geometric relativistic phase from Lorentz symmetry breaking effects in the cosmic string spacetime

Belli, P., Results and strategies in dark matter detection

Bellorín, J., Restuccia, A. \& Sotomayor, A., Solutions with throats in Hořava
25 (2016) 1650048

25 (2016) 1630021

25 (2016) 1644005

25 (2016) 1642008

25 (2016) 1675001

25 (2016) 1630023

25 (2016) 1630016

25 (2016) 1642012

25 (2016) 1650087

25 (2016) 1641003

25 (2016) 1630013 gravity with cosmological constant

Belvedere, G., see Del Popolo

Benetti, M., see Burigana

Benone, C. L., Stationary configurations around charged rotating black holes

Bera, S., see Banerjee

Berenstein, D. \& Miller, A., Reconstructing spacetime from the hologram, even in the classical limit, requires physics beyond the Planck scale

Berkowitz, E., Hanada, M. \& Maltz, J., A microscopic description of black hole evaporation via holography

Bernabei, R., Particle dark matter direct detection

Bernar, R. P., Crispino, L. C. B. \& Higuchi, A., Graviton two-point function in $3+1$ static de Sitter spacetime

Berti, E., Cardoso, V., Crispino, L. C. B., Gualtieri, L., Herdeiro, C. \& Sperhake, U., Numerical relativity and high energy physics: Recent developments

Berti, E., see Silva

Bessa, C. H. G., Bezerra, V. B. \& Silva, J. C. J., Casimir effect in spacetimes with cosmological constant

Bezerra, V. B., Mota, H. F. \& Muniz, C. R., Remarks on a gravitational analogue of the Casimir effect

Bezerra, V. B., see Bessa

Bhar, P., see Singh

Bi, X.-J., see Zhao

Blau, M. \& O'Loughlin, M., Horizon shells: Classical structure at the horizon of a black hole

Bogadi, R. S., see Govender

Bojowald, M., Symmetries of spacetime
25 (2016) 1650016

25 (2016) 1650093

25 (2016) 1630016

25 (2016) 1641012

25 (2016) 1644005

25 (2016) 1644012

25 (2016) 1644002

25 (2016) 1630018

25 (2016) 1641016

25 (2016) 1641022

25 (2016) 1641006

25 (2016) 1641017

25 (2016) 1641018

25 (2016) 1641017

25 (2016) 1650099

25 (2016) 1650006

25 (2016) 1644010

25 (2016) 1650037

25 (2016) 1644007 
Cumulative author index

Boko, R. D., Houndjo, M. J. S. \& Tossa, J., Stability and phase space analysis in $f(R)$ theory with generalized exponential $f(R)$ model

Bolliet, B., see Barrau

Bolotin, Yu. L., Cherkaskiy, V. A. \& Lemets, O. A., New cosmographic constraints on the dark energy and dark matter coupling

Bonder, Y., Torsion or not torsion, that is the question

Boonserm, P., Ngampitipan, T. \& Visser, M., Mimicking static anisotropic fluid spheres in general relativity

Borowiec, A., see Sporea

Brihaye, Y. \& Ducobu, L., Black holes with scalar hair in Einstein-GaussBonnet gravity

Bronnikov, K. A., Fabris, J. C. \& Rodrigues, D. C., On black hole structures in scalar-tensor theories of gravity

Buchert, T., Coley, A. A., Kleinert, H., Roukema, B. F. \& Wiltshire, D. L., Observational challenges for the standard FLRW model

Buettner, D. J., see Morley

Burigana, C., Battistelli, E. S., Benetti, M., Cabass, G., De Bernardis, P., Di Serego Alighieri, S., Di Valentino, E., Gerbino, M., Giusarma, E., Gruppuso, A., Liguori, M., Masi, S., NorgaardNielsen, H. U., Rosati, P., Salvati, L., Trombetti, T. \& Vielva, P., Recent results and perspectives on cosmology and fundamental physics from microwave surveys

Burigana, C., see Battistelli

Burigana, C., see Massardi

Cabass, G., see Burigana
25 (2016) 1650098

25 (2016) 1642008

25 (2016) 1650056

25 (2016) 1644013

25 (2016) 1650019

25 (2016) 1650043

25 (2016) 1650084

25 (2016) 1641005

25 (2016) 1630007

25 (2016) 1650089

25 (2016) 1630016

25 (2016) 1630023

25 (2016) 1640009

25 (2016) 1630016
Cabral, L. A., see Seidel

Cai, R.-G. \& Yang, R.-Q., Understanding strongly coupling magnetism from holographic duality

Calcagni, G., Modesto, L. \& Nardelli, G., Quantum spectral dimension in quantum field theory

Caldwell, R., Cosmic parity violation due to a flavorspace locked gauge field

Canfora, F., see Ayón-Beato

Cao, S., see Pan

Cao, Z., see $\mathrm{He}$

Cao, Z., see He

Capozziello, S., Luongo, O. \& Paolella, M., Bounding $f(R)$ gravity by particle production rate

Cardoso, V., see Berti

Cardoso, V., see Oliveira

Carlip, S., Spontaneous dimensional reduction in quantum gravity

Carot, J., see Herrera

Casadio, R., Giugno, A. \& Micu, O., Horizon quantum mechanics: A hitchhiker's guide to quantum black holes

Casals, M., A self-force approach to the two-body problem: The Green function method

Chakraborty, S. \& Lochan, K., Quantum leaps of black holes: Magnifying glasses of quantum gravity

Chakraborty, S., see Dutta

Chakraborty, S., see Dutta

Chakraborty, S., see Dutta

Chen, C.-C., see Nam

Chen, C.-H., see Nam

Chen, C.-M., see Nester

Chen, C.-W., see Nam

Chen, J.-H., see Guo

Chen, P., Chiang, H.-W. \& Hu, Y.-C., A quantized spacetime based on $\operatorname{Spin}(3,1)$ symmetry

Chen, P., Ong, Y. C., Page, D. N., Sasaki, M. \& Yeom,
25 (2016) 1645011

25 (2016) 1641007

25 (2016) 1650058

25 (2016) 1640011

25 (2016) 1641009

25 (2016) 1650003

25 (2016) 1650039

25 (2016) 1650086

25 (2016) 1630010

25 (2016) 1641022

25 (2016) 1641019

25 (2016) 1643003

25 (2016) 1650036

25 (2016) 1630006

25 (2016) 1641015

25 (2016) 1644024

25 (2016) 1650110

25 (2016) 1675002

25 (2016) 1650051

25 (2016) 1645013

25 (2016) 1645013

25 (2016) 1645002

25 (2016) 1645013

25 (2016) 1650021

25 (2016) 1645004 
D.-H., Unclothed firewalls

25 (2016) 1645003

Chen, P., see Nam

Chen, P.-N., Wang, M.-T. \& Yau, S.-T., Quasi-local energy in presence of gravitational radiation

Chen, Y.-C., see Nam

Cherkaskiy, V. A., see Bolotin

Chiang, H.-W., see Chen

Christmann, M. H. B., see Jantsch

Cianfrani, F., see Alesci

Codello, A. \& Jain, R. K., Leading gravitational corrections and a unified universe

Coelho, F. S. \& Sampaio, M. O. P., Radiation from a $D$-dimensional collision of shock waves: Twodimensional reduction and Carter-Penrose diagram

Coelho, J. G., see Lobato

Coley, A. A., see Buchert

Colin, S. \& Valentini, A., Robust predictions for the large-scale cosmological power deficit from primordial quantum nonequilibrium

Compère, G., Bulk supertranslation memories: A concept reshaping the vacua and black holes of general relativity

Contaldi, C. R., Imaging cosmic polarization rotation

Contopoulos, I. G., Esposito, F. P., Kleidis, K., Papadopoulos, D. B. \& Witten, L., Generating solutions to the Einstein field equations

Cook, W. G., Figueras, P., Kunesch, M., Sperhake, U. \& Tunyasuvunakool, S., Dimensional reduction in numerical relativity: Modified Cartoon formalism and regularization
25 (2016) 1645013

25 (2016) 1645001

25 (2016) 1645013

25 (2016) 1650056

25 (2016) 1645004

25 (2016) 1650031

25 (2016) 1642005

25 (2016) 1644023

25 (2016) 1641010

25 (2016) 1641025

25 (2016) 1630007

25 (2016) 1650068

25 (2016) 1644006

25 (2016) 1640014

25 (2016) 1650022

25 (2016) 1641013
Cooperstock, F. I., The power of weak-field GR gravity

Corichi, A. \& Karami, A., Loop quantum cosmology of Bianchi IX: Inclusion of inverse triad corrections

Corichi, A., RubalcavaGarcía, I. \& Vukašinac, T., Actions, topological terms and boundaries in firstorder gravity: A review

Craig, D. A., The consistent histories approach to loop quantum cosmology

Crispino, L. C. B., see Bernar

Crispino, L. C. B., see Berti

Crispino, L. C. B., see Leite

Crispino, L. C. B., see Macedo

Crispino, L. C. B., see Oliveira

Crispino, L. C. B., see de Lima

Cruz, C. N., On the electrodynamics of moving particles in a quasi flat spacetime with Lorentz violation and its cosmological implications

Cunha, P. V. P., Herdeiro, C. A. R., Radu, E. \& Rúnarsson, H. F., Shadows of Kerr black holes with and without scalar hair

Darabi, F., Felegary, F. \& Setare, M. R., Cosmological dynamics of interacting logarithmic entropy corrected holographic dark energy model

Das, S., see Ali

Das, S., see Mamon

De Bernardis, P., see Burigana

de Bernardis, P., see Battistelli

de Bernardis, P. \& Masi, S., Cosmic microwave background and cosmic polarization rotation: An experimentalist view
25 (2016) 1644017

25 (2016) 1642011

25 (2016) 1630011

25 (2016) 1642009

25 (2016) 1641016

25 (2016) 1641022

25 (2016) 1641024

25 (2016) 1641008

25 (2016) 1641019

25 (2016) 1641002

25 (2016) 1650096

25 (2016) 1641021

25 (2016) 1650104

25 (2016) 1644001

25 (2016) 1650032

25 (2016) 1630016

25 (2016) 1630023

25 (2016) 1640012 
Dehghani, M. H., see Sheykhi

de Lima, M. C. \& Crispino, L. C. B., Crommelin's and Davidson's visit to Amazonia and the 1919 total solar eclipse

Del Popolo, A., Saburova, A. \& Belvedere, G., The inner structure of dark matter haloes in the Hubble sequence

Dempsey, D. \& Dolan, S. R., Waves and null congruences in a draining bathtub

Deng, X.-M., The second post-Newtonian light propagation and its astrometric measurement in the Solar System: Light time and frequency shift

Denisov, V. I., Ilyina, V. A. \& Sokolov, V. A., Nonlinear vacuum electrodynamics influence on the spacetime structure and massive particles orbits properties in Einstein-BornInfeld theory

de Oliveira, E. S., see Macedo

de Oliveira, E. S., see Leite

Di Prisco, A., see Herrera

Di Serego Alighieri, S., see Burigana

Di Valentino, E., see Burigana

Di Virgilio, A., see Lämmerzahl

Dichiara, S., see Frontera

Dirkes, A. R. P., Maziashvili, M. \& Silagadze, Z. K., Black hole remnants due to Planck-length deformed QFT

Dog̃ru, M. U., see Taṣer

Dolan, S. R., see Dempsey

Dolan, S. R., see Leite

Domènech, G. \& Sasaki, M., Conformal frames in cosmology

Ducobu, L., see Brihaye
25 (2016) 1650018

25 (2016) 1641002

25 (2016) 1650093

25 (2016) 1641004

25 (2016) 1650082

25 (2016) 1640003

25 (2016) 1641008

25 (2016) 1641024

25 (2016) 1650036

25 (2016) 1630016

25 (2016) 1630016

25 (2016) 1630022

25 (2016) 1630014

25 (2016) 1650015

25 (2016) 1650017

25 (2016) 1641004

25 (2016) 1641024

25 (2016) 1645006 25 (2016) 1650084
Dutta, S. \& Chakraborty, S., A study of phantom scalar field cosmology using Lie and Noether symmetries

Dutta, S. \& Chakraborty, S., Answer to the comments on the paper "A study of phantom scalar field cosmology using Lie and Noether symmetries"

Dutta, S., Lakshmanan, M. \& Chakraborty, S., Quintom cosmological model and some possible solutions using Lie and Noether symmetries

Ebadi, H., see Moradpour

Elizaga Navascués, B., Martín-Benito, M. \& Mena Marugán, G. A., Hybrid models in loop quantum cosmology

Ellis, J., No-scale supergravity inflation: A bridge between string theory and particle physics?

Ellis, J., Time to move on?

Emparan, R. \& Mar, M.tínez Black hole fusion made easy

Engelhardt, N. \& Horowitz, G. T., New insights into quantum gravity from gauge/gravity duality

Engle, J. S., see Beetle

Esguerra, J. P., see Acuña

Esposito, F. P., see Contopoulos

Fabris, J. C., see Bronnikov

Faizal, M. \& Kruglov, S. I., Deformation of the Dirac equation

Faizal, M., see Hammad

Farahani, H., see Sadeghi

Farhangkhah, N., New solutions of exotic charged black holes and their stability

Farinelli, R., see Frontera

Fatima, H. I., see Sharif

Fatima, H. I., see Sharif

Fedotov, A. M. \& Narozhny, N. B., Scalar and fermion representations
25 (2016) 1650051

25 (2016) 1675002

25 (2016) 1650110

25 (2016) 1650014

25 (2016) 1642007

25 (2016) 1630027

25 (2016) 1645014

25 (2016) 1644015

25 (2016) 1643002

25 (2016) 1642012

25 (2016) 1650001

25 (2016) 1650022

25 (2016) 1641005

25 (2016) 1650013

25 (2016) 1650080

25 (2016) 1650108

25 (2016) 1650030

25 (2016) 1630014

25 (2016) 1650011

25 (2016) 1650083 
of the Lorentz group in Minkowski plane, QFT correlators, pair creation in electric field and the Unruh effect

Felegary, F., see Darabi

Ferrari, V., see Pani

Fewster, C. J., Locally covariant quantum field theory and the spinstatistics connection

Figueras, P., see Cook

Firouzjaee, J. T., see Allahyari

Fischler, W. \& Kundu, S., Physical effects of the gravitational $\Theta$-parameter

Fischler, W., Jimmy \& Lorshbough, D., Decoupled sectors and Wolf-Rayet galaxies

Frontera, F., Amati, L., Farinelli, R., Dichiara, S., Guidorzi, C., Landi, R. \& Titarchuk, L., Possible physical explanation of the intrinsic $E_{p, i^{-}}$ "intensity" correlation commonly used to "standardize" GRBs

Galluzzi, V. \& Massardi, M., The polarimetric multifrequency radio sources properties

Galluzzi, V., see Massardi

Gambini, R., Olmedo, J. \& Pullin, J., Schrödingerlike quantum dynamics in loop quantized black holes

Gao, Z. F., see Li

García-Berro, E., Kilic, M. \& Kepler, S. O., Magnetic white dwarfs: Observations, theory and future prospects

Gasperini, M., Do alien particles exist, and can they be detected?

Gerbino, M., see Burigana

Ghaffari, S., see Sheykhi

Giddings, S. B., Observational strong gravity and quantum black hole structure
Giugno, A., see Casadio

Giusarma, E., see Burigana

Gogberashvili, M., Mantidze, I., Sakhelashvili, O.

25 (2016) 1630008

25 (2016) 1650104

25 (2016) 1641001

25 (2016) 1630015

25 (2016) 1641013

25 (2016) 1650005

25 (2016) 1644022

25 (2016) 1650094

25 (2016) 1630014

25 (2016) 1640005

25 (2016) 1640009

25 (2016) 1642006

25 (2016) 1650002

25 (2016) 1630005

25 (2016) 1630030

25 (2016) 1630016

25 (2016) 1650018

25 (2016) 1644014

\& Shengelia, T., Standing waves braneworlds

Golda, Z. A., see Sokołowski

Gorji, M. A., see Vakili

Govender, M., Bogadi, R. S., Lortan, D. B. \& Maharaj, S. D., Radiating collapse in the presence of anisotropic stresses

Govender, M., see Naidu

Grain, J., The perturbed universe in the deformed algebra approach of loop quantum cosmology

Gruppuso, A., see Burigana

Gruppuso, A., Cosmological birefringence constraints from the Planck 2015 CMB likelihood

Gualtieri, L., see Berti

Gualtieri, L., see Pani

Gubitosi, G., see AmelinoCamelia

Gubitosi, G., Disentangling birefringence from standard physics in CMB measurements and distinguishing among production mechanisms

Guendelman, E., Nissimov, E. \& Pacheva, S., Gravityassisted emergent Higgs mechanism in the postinflationary epoch

Guidorzi, C., see Frontera

Guo, E.-D., Li, M. \& Sun, J.-R., CFT dual of charged AdS black hole in the large dimension limit

Guo, H., Shu, F.-W., Chen, J.-H., Li, H. \& Yu, Z., A holographic model of $d$-wave superconductor vortices with Lifshitz scaling

Haggard, H. M. \& Rovelli, C., Quantum gravity effects around Sagittarius A*

Hajkhalili, S., see Sheykhi
25 (2016) 1630006

25 (2016) 1630016

25 (2016) 1630019

25 (2016) 1650007

25 (2016) 1650028

25 (2016) 1650037

25 (2016) 1650092

25 (2016) 1642003

25 (2016) 1630016

25 (2016) 1640007

25 (2016) 1641022

25 (2016) 1641001

25 (2016) 1650027

25 (2016) 1640006

25 (2016) 1644008

25 (2016) 1630014

25 (2016) 1650085

25 (2016) 1650021
25 (2016) 1644021

25 (2016) 1650062 
Halzen, F., Icecube and the discovery of high-energy cosmic neutrinos

Hammad, F. \& Faizal, M., A logarithmic correction in the entropy functional formalism

Hammad, F., Conformal mapping of the Misner-Sharp mass from gravitational collapse

Hammond, R. T., Stringy Maxwell charge and the magnetic dipole moment

Hanada, M., see Berkowitz

He, X. \& Cao, Z., Hertz potential formalism for force-free electrodynamics and its application to Brennan-Gralla-Jacobson solutions

He, X., Cao, Z. \& Jing, J., Asymptotical null structure of an electro-vacuum spacetime with a cosmological constant

Hehl, F. W., Itin, Y. \& Obukhov, Y. N., On Kottler's path: Origin and evolution of the premetric program in gravity and in electrodynamics

Hehl, F. W., Axion and dilaton + metric emerge jointly from an electromagnetic model universe with local and linear response behavior

Heidenreich, B., Reece, M. \& Rudelius, T., Axion experiments to algebraic geometry: Testing quantum gravity via the Weak Gravity Conjecture

Hendi, S. H., Panahiyan, S. \& Momennia, M., Extended phase space of AdS black holes in Einstein-Gauss-Bonnet gravity with a quadratic nonlinear electrodynamics

Hendi, S. H., Panahiyan, S. \& Panah, B. E., P-
$V$ criticality and geometrical thermodynamics of black holes with BornInfeld type nonlinear electrodynamics

25 (2016) 1650080

Herdeiro, C. A. R., Radu, E. \& Rúnarsson, H. F., Spinning boson stars and Kerr black holes with scalar hair: The effect of selfinteractions

Herdeiro, C. A. R., see Cunha

25 (2016) 1640001

25 (2016) 1644002

25 (2016) 1650039

25 (2016) 1650086

25 (2016) 1640016

25 (2016) 1640015

25 (2016) 1643005

25 (2016) 1650063
Herdeiro, C., see Berti

Herrera, L., Di Prisco, A., Ospino, J. \& Carot, J., Axially symmetric dissipative fluids in the quasistatic approximation

Higuchi, A., see Bernar

Hogan, M. E., see Beetle

Holdom, B. \& Ren, J., Quadratic gravity: From weak to strong

Hořava, P., Surprises with nonrelativistic naturalness

Horowitz, G. T., see Engelhardt

Hossain, M. I., see Hossain

Hossain, M. J., Rahman, M. A. \& Hossain, M. I., Bekenstein-Hawking entropy by energy quantization from ReissnerNordström black hole

Houndjo, M. J. S., see Boko

Houndjo, M. J. S., see Salako

Hsu, S.-Y., see Nam

$\mathrm{Hu}$, Y.-C., see Chen

Huang, C.-G., see Wang

Huang, J.-J., see Nam

Huang, M.-H. A., see Nam

Huang, X.-L., Xu, Y., Liu, C.-Z. \& Liu, G.-Z., Effects of tensor couplings of $\omega$ and $\rho$ mesons on ${ }^{1} S_{0}$ nucleon superfluidity in neutron star matter

Huang, Y.-C., see Sun

Ibungochouba Singh, T., Ablu Meitei, I. \& Yugindro Singh, K., Quantum radiation of Maxwell's electromagnetic field in
25 (2016) 1650010

25 (2016) 1641014

25 (2016) 1641021

25 (2016) 1641022

25 (2016) 1650036

25 (2016) 1641016

25 (2016) 1642012

25 (2016) 1643004

25 (2016) 1645007

25 (2016) 1643002

25 (2016) 1650034

25 (2016) 1650034

25 (2016) 1650098

25 (2016) 1650076

25 (2016) 1645013

25 (2016) 1645004

25 (2016) 1650100

25 (2016) 1645013

25 (2016) 1645013

25 (2016) 1650059

25 (2016) 1650038 
nonstationary Kerr-de Sitter black hole

Ilyina, V. A., see Denisov

Imbiriba, B. C. O., Spacelike gravitational radiation extraction from rotating binary black holes

Iqbal, A., see Jawad

Ishaque, O., see Ahmed

Itin, Y., see Hehl

Ivanov, A. A. \& Timofeev, L. V., Temporal signatures of the Cherenkov light induced by extensive air showers of cosmic rays detected with the Yakutsk array

Ivanov, A. A., Testing for uniformity of ultra-high energy cosmic ray arrival directions

Ivanov, B. V., A different approach to anisotropic spherical collapse with shear and heat radiation

Jain, R. K., see Codello

Jalalzadeh, S., Rostami, T. \& Moniz, P. V., Quantum cosmology: From hidden symmetries towards a new (supersymmetric) perspective

Jamil, M., see Babar

Jantsch, R. C. S., Christmann, M. H. B. \& Kremer, G. M., The van der Waals fluid and its role in cosmology

Jawad, A. \& Iqbal, A., Viscous Chaplygin gas models as spherical top-hat collapsing fluids

Jawad, A., see Rani

Jawad, A., see Salako

Jawad, A., see Salako

Jia, H.-Y., see Zhao

Jimmy, see Fischler

Jing, J., see He

Joshi, P. S., see Satin

Kamali, A. D. \& Sabounchi, P., A note on Planck scale corrections to bouncing universe

Kang, K., see Wong
25 (2016) 1650061

25 (2016) 1640003

25 (2016) 1641023

25 (2016) 1650074

25 (2016) 1650035

25 (2016) 1640016

25 (2016) 1650090

25 (2016) 1650065

25 (2016) 1650049

25 (2016) 1644023

25 (2016) 1630009

25 (2016) 1650024

25 (2016) 1650031

25 (2016) 1650074

25 (2016) 1650102

25 (2016) 1650055

25 (2016) 1650076

25 (2016) 1650006

25 (2016) 1650094

25 (2016) 1650086

25 (2016) 1650023

25 (2016) 1650046 25 (2016) 1645012
Karami, A., see Corichi

Karchi, A. P. K. \& Shojaie, H., An FLRW cosmology with a chameleon field

Kaufman, J., Leon, D. \& Keating, B., Using the Crab Nebula as a high precision calibrator for cosmic microwave background polarimeters

Keating, B., see Kaufman

Kepler, S. O., see GarcíaBerro

Khurshudyan, M., see Sadeghi

Kilic, M., see García-Berro

Kim, S. P., Schwinger effect, Hawking radiation and Unruh effect

Kirillov, A. A. \& Savelova, E. P., Cosmological wormholes

Kirillov, A. A., see Battistelli

Kleidis, K., see Contopoulos

Kleinert, H., see Buchert

Kramer, M., Pulsars as probes of gravity and fundamental physics

Kremer, G. M. \& André, R., Analysis of instability of systems composed by dark and baryonic matter

Kremer, G. M., see Jantsch

Kruglov, S. I., see Faizal

Kruglov, S. I., Acceleration of universe by nonlinear electromagnetic fields

Kubeka, A. S., see Sadeghi

Kundu, S., see Fischler

Kunesch, M., see Cook

Lämmerzahl, C. \& Di Virgilio, A., Experimental gravitation

Lakshmanan, M., see Dutta

Landi, R., see Frontera

Landim, R. C. G., Holographic dark energy from minimal supergravity

Landry, A. \& Paranjape, M. B., Graviton laser

Lee, J., see Overduin

Lee, T. H., Induced dark energy in a warped
25 (2016) 1642011

25 (2016) 1650045

25 (2016) 1640008

25 (2016) 1640008

25 (2016) 1630005

25 (2016) 1650108

25 (2016) 1630005

25 (2016) 1645005

25 (2016) 1650075

25 (2016) 1630023

25 (2016) 1650022

25 (2016) 1630007

25 (2016) 1630029

25 (2016) 1650012

25 (2016) 1650031

25 (2016) 1650013

25 (2016) 1640002

25 (2016) 1650077

25 (2016) 1644022

25 (2016) 1641013

25 (2016) 1630022

25 (2016) 1650110

25 (2016) 1630014

25 (2016) 1650050

25 (2016) 1644016

25 (2016) 1650069 
Cumulative author index

braneworld and accelerating universe

Leite, L. C. S., Crispino, L. C. B., de Oliveira, E. S., Macedo, C. F. B. \& Dolan, S. R., Absorption of massless scalar field by rotating black holes

Lemets, O. A., see Bolotin Leon, D., see Kaufman

Li, H., see Guo

Li, L., see Pan

Li, M., see Guo

Li, X. D., see Li

Li, X. H., Gao, Z. F., Li, X. D., Xu, Y., Wang, P., Wang, N. \& Peng, Q. H., Numerically fitting the electron Fermi energy and the electron fraction in a neutron star

Li, Z., see Yang

Liguori, M., see Burigana

Lim, Y.-K., see Babar

Liu, C.-Z., see Huang

Liu, G.-Z., see Huang

Liu, T.-C., see Nam

Lobato, R. V., Malheiro, M. \& Coelho, J. G., Magnetars and white dwarf pulsars

Lobo, F. S. N., From the Flamm-Einstein-Rosen bridge to the modern renaissance of traversable wormholes

Lochan, K., see Chakraborty

López-Corredoira, M., Melia, F., Lusso, E. \& Risaliti, G., Cosmological test with the QSO Hubble diagram

Lorshbough, D., see Fischler

Lortan, D. B., see Govender

Luongo, O., see Capozziello

Lusso, E., see LópezCorredoira

Macedo, C. F. B., Crispino, L. C. B. \& de Oliveira, E. S., Scalar waves in regular Bardeen black holes: Scattering, absorption and quasinormal modes

Macedo, C. F. B., see Leite
25 (2016) 1650004

25 (2016) 1641024

25 (2016) 1650056

25 (2016) 1640008

25 (2016) 1650021

25 (2016) 1650003

25 (2016) 1650085

25 (2016) 1650002

25 (2016) 1650002

25 (2016) 1650026

25 (2016) 1630016

25 (2016) 1650024

25 (2016) 1650059

25 (2016) 1650059

25 (2016) 1645013

25 (2016) 1641025

25 (2016) 1630017

25 (2016) 1644024

25 (2016) 1650060

25 (2016) 1650094

25 (2016) 1650037

25 (2016) 1630010

25 (2016) 1650060

25 (2016) 1641008

25 (2016) 1641024
Maeda, K. \& Terada, Y., Progenitors of type Ia supernovae

Maharaj, S. D., see Govender

Malafarina, D., see Satin

Malheiro, M., see Lobato

Maltz, J., see Berkowitz

Mamon, A. A. \& Das, S., A divergence-free parametrization of deceleration parameter for scalar field dark energy

Mannheim, P. D., Conformal invariance and the metrication of the fundamental forces

Mansouri, R., see Allahyari

Mantidze, I., see Gogberashvili

Mar, M.tínez, see Emparan

Martín-Benito, M., see Elizaga Navascués

Maselli, A., see Pani

Maselli, A., see Silva

Masi, S., see Battistelli

Masi, S., see Burigana

Masi, S., see de Bernardis

Massardi, M., Galluzzi, V., Paladino, R. \& Burigana, C., Polarization of extragalactic radio sources: CMB foregrounds and telescope calibration issues

Massardi, M., see Galluzzi

Mathur, S. D., What prevents gravitational collapse in string theory?

Maziashvili, M., see Dirkes

McDevitt, T., see Stuckey

Melia, F., see LópezCorredoira

Mena Marugán, G. A., see Elizaga Navascués

Mendonça, P., see Beetle

Mercati, F., Quantum $\kappa$ deformed differential geometry and field theory

Micu, O., see Casadio

Miller, A., see Berenstein

Minamitsuji, M., see Silva

Modesto, L., see Calcagni

Momennia, M., see Hendi

Moniz, P. V., see Jalalzadeh
25 (2016) 1630024

25 (2016) 1650037

25 (2016) 1650023

25 (2016) 1641025

25 (2016) 1644002

25 (2016) 1650032

25 (2016) 1644003

25 (2016) 1650005

25 (2016) 1630019

25 (2016) 1644015

25 (2016) 1642007

25 (2016) 1641001

25 (2016) 1641006

25 (2016) 1630023

25 (2016) 1630016

25 (2016) 1640012

25 (2016) 1640009

25 (2016) 1640005

25 (2016) 1644018

25 (2016) 1650015

25 (2016) 1644004

25 (2016) 1650060

25 (2016) 1642007

25 (2016) 1642012

25 (2016) 1650053

25 (2016) 1630006

25 (2016) 1644012

25 (2016) 1641006

25 (2016) 1650058

25 (2016) 1650063

25 (2016) 1630009 
Moon, T., see Myung

Moradpour, H., Abri, A. \& Ebadi, H., Thermodynamic behavior and stability of Polytropic gas

Moraes, P. H. R. S., Cosmic acceleration from varying masses in five dimensions

Morley, P. D. \& Buettner, D. J., A dark matter signature for condensed neutrinos

Moschella, U., Infrared surprises in the de Sitter universe

Mota, H. F., see Bezerra

Muniz, C. R., see Bezerra

Myrzakul, S., Myrzakulov, R. \& Sebastiani, L., $f(\phi) R$-models for inflation

Myrzakulov, R., see Myrzakul

Myrzakulov, R., see Sami

Myung, Y. S., Moon, T. \& Park, Y.-J., Einsteinsingleton theory and its power spectra in de Sitter inflation

Naidu, N. F. \& Govender, M., The influence of initial conditions during dissipative collapse

Najafi, T., see Abdolmaleki

Nam, J. W., Chen, C.-C., Chen, C.-H., Chen, C.W., Chen, P., Chen, Y.C., Hsu, S.-Y., Huang, J.J., Huang, M.-H. A., Liu, T.-C., Ř́pa, J., Shiao, Y.S., Wang, M.-Z. \& Wang, S.-H., Design and implementation of the TAROGE experiment

Nardelli, G., see Calcagni

Narozhny, N. B., see Fedotov

Nashed, G. G. L., Rotating black holes in the teleparallel equivalent of general relativity

Nawarajan, D. \& Visser, M., Global properties of physically interesting Lorentzian spacetimes
25 (2016) 1650107

25 (2016) 1650014

25 (2016) 1650009

25 (2016) 1650089

25 (2016) 1641020

25 (2016) 1641018

25 (2016) 1641018

25 (2016) 1650041

25 (2016) 1650041

25 (2016) 1630031

25 (2016) 1650107

25 (2016) 1650092

25 (2016) 1650040

25 (2016) 1645013

25 (2016) 1650058

25 (2016) 1630008

25 (2016) 1650079

25 (2016) 1650106
Nester, J. M. \& Chen, C.M., Gravity: A gauge theory perspective

Neto, G. B. L., see Battistelli

Neznamov, V. P. \& Safronov, I. I., The effective method to calculate eigenvalues of Chandrasekhar-Page angular equations

Ngampitipan, T., see Boonserm

25 (2016) 1645002

25 (2016) 1630023

25 (2016) 1650091

25 (2016) 1650019

Ni, W.-T., A nonmetric theory of gravity

Ni, W.-T., Equivalence principles, spacetime structure and the cosmic connection

Ni, W.-T., Genesis of general relativity - A concise exposition

Ni, W.-T., Gravitational wave detection in space

Ni, W.-T., On spacetime structure and electrodynamics

Ni, W.-T., Solar-system tests of the relativistic gravity

Nissimov, E., see Guendelman

25 (2016) 1640017

25 (2016) 1630002

25 (2016) 1630004

25 (2016) 1630001

25 (2016) 1603001

25 (2016) 1630003

25 (2016) 1644008

Nomoto, K., First stars, hypernovae, and superluminous supernovae

Norgaard-Nielsen, H. U., see Battistelli

Norgaard-Nielsen, H. U., see Burigana

Nozari, K., see Behboodi

Nunes, R. C., Connecting inflation with late cosmic acceleration by particle production

Obukhov, Y. N., see Hehl

Oikonomou, V. K., A note on Schwarzschild-de Sitter black holes in mimetic $F(R)$ gravity

Oliveira, L. A., Cardoso, V. \& Crispino, L. C. B., Superresonant instability of a compressible hydrodynamic vortex

Olmedo, J., see Gambini

Olmedo, J., Evolution in totally constrained models: Schrödinger vs. Heisenberg pictures
25 (2016) 1630025

25 (2016) 1630023

25 (2016) 1630016

25 (2016) 1650087

25 (2016) 1650067

25 (2016) 1640016

25 (2016) 1650078

25 (2016) 1641019

25 (2016) 1642006

25 (2016) 1642004 
O’Loughlin, M., see Blau

Ong, Y. C., see Chen

Ospino, J., see Herrera

Ostermann, P., see Battistelli

Otalora, G., A novel teleparallel dark energy model

Overduin, J., Prins, N. \& Lee, J., Supernova constraints on higherdimensional cosmology with a phantom field

Pacheva, S., see Guendelman

Padmanabhan, T., The atoms of space, gravity and the cosmological constant

Page, D. N., see Chen

Paladino, R., see Massardi

Paliathanasis, A., Basilakos, S. \& Tsamparlis, M., Comment on "A study of phantom scalar field cosmology using Lie and Noether symmetries"

Palmisano, G., see AmelinoCamelia

Pan, Y., Cao, S. \& Li, L., Constraints on interacting dark energy from time delay lenses

Panah, B. E., see Hendi

Panahiyan, S., see Hendi

Panahiyan, S., see Hendi

Pani, P., Gualtieri, L., Maselli, A. \& Ferrari, V., Recent developments in the tidal deformability of spinning compact objects

Pant, N., see Singh

Paolella, M., see Capozziello

Papadopoulos, D. B., see Contopoulos

Parameswaran, S. L. \& Zavala, I., Prospects for primordial gravitational waves in string inflation

Paranjape, M. B., see Landry

Park, Y.-J., see Myung

Pawłowski, T., Universe's memory and spontaneous coherence in loop quantum cosmology

Peña, F., see Balart

Peng, Q. H., see Li
25 (2016) 1644010

25 (2016) 1645003

25 (2016) 1650036

25 (2016) 1630023

25 (2016) 1650025

25 (2016) 1650069

25 (2016) 1644008

25 (2016) 1630020

25 (2016) 1645003

25 (2016) 1640009

25 (2016) 1675001

25 (2016) 1650027

25 (2016) 1650003

25 (2016) 1650010

25 (2016) 1650010

25 (2016) 1650063

25 (2016) 1641001

25 (2016) 1650099

25 (2016) 1630010

25 (2016) 1650022

25 (2016) 1644011

25 (2016) 1644016

25 (2016) 1650107

25 (2016) 1642013

25 (2016) 1650072

25 (2016) 1650002
Pilipenko, S. V., see Arkhipova

25 (2016) 1650109

Piran, T., The quantum interaction of macroscopic objects and gravitons

Pourhassan, B., see Sadeghi

Prins, N., see Overduin

Pshirkov, M. S., Prospects for strangelet detection with large-scale cosmic ray observatories

Pullin, J., see Gambini

Qiang, L.-E. \& Xu, P., Probing the postnewtonian physics of semi-conservative metric theories through secular tidal effects in satellite gradiometry missions

Radu, E., see Cunha

Radu, E., see Herdeiro

Rahman, M. A., see Hossain

Rani, S. \& Jawad, A., Cosmological implications of DGP braneworld via wellknown holographic dark energy models

Ranjit, C. \& Rudra, P., Study of some cosmological parameters for interacting new holographic dark energy model in $f(T)$ gravity

Reece, M., see Heidenreich

Rehman, M. U., see Ahmed

Rejzner, K., Effective quantum gravity observables and locally covariant QFT

Ren, J., see Holdom

Restuccia, A., see Bellorín

Reyes, I., see Bañados

Řípa, J., see Nam

Risaliti, G., see LópezCorredoira

Rodrigues, D. C., see Bronnikov

Roman, M., see Battistelli

Rosati, P., see Battistelli

Rosati, P., see Burigana

Rossetti, M., see Battistelli

Rostami, M., see Sadeghi

Rostami, T., see Jalalzadeh

Roukema, B. F., see Buchert

Rovelli, C., see Haggard
25 (2016) 1644020

25 (2016) 1650077

25 (2016) 1650069

25 (2016) 1650103

25 (2016) 1642006

25 (2016) 1650070

25 (2016) 1641021

25 (2016) 1641014

25 (2016) 1650034

25 (2016) 1650102

25 (2016) 1650008

25 (2016) 1643005

25 (2016) 1650035

25 (2016) 1630012

25 (2016) 1643004

25 (2016) 1650016

25 (2016) 1630021

25 (2016) 1645013

25 (2016) 1650060

25 (2016) 1641005

25 (2016) 1630023

25 (2016) 1630023

25 (2016) 1630016

25 (2016) 1630023

25 (2016) 1650077

25 (2016) 1630009

25 (2016) 1630007

25 (2016) 1644021 
Rubalcava-García, I., see Corichi

Rudelius, T., see Heidenreich

Rudra, P., see Ranjit

Ruggiero, M. L., Light bending in $f(T)$ gravity

Rúnarsson, H. F., see Herdeiro

Rúnarsson, H. F., see Cunha

Sabounchi, P., see Kamali

Saburova, A., see Del Popolo

Sadeghi, J., Khurshudyan, M. \& Farahani, H., Interacting ghost dark energy models in the higher dimensional cosmology

Sadeghi, J., Pourhassan, B., Kubeka, A. S. \& Rostami, M., Logarithmic corrected polynomial $f(R)$ inflation mimicking a cosmological constant

Safronov, I. I., see Neznamov

Sahraee, M. \& Setare, M. R., The effect of backreaction on inflationary BransDicke cosmology

Sakhelashvili, O., see Gogberashvili

Salako, I. G. \& Jawad, A., Superresonance phenomenon from acoustic black holes in neoNewtonian theory

Salako, I. G., Houndjo, M. J. S. \& Jawad, A., Generalized Mattig's relation in Brans-Dicke-Rastall gravity

Salehi, A. \& Aftabi, S., An approach to investigate stability, cosmography and the dark energy dipole: A case study of cosmological models in $R^{1+\epsilon}$

Salvati, L., see Burigana

Sami, M. \& Myrzakulov, R., Late-time cosmic acceleration: $\mathrm{ABCD}$ of dark energy and modified theories of gravity
Sampaio, M. O. P., see 25 (2016) 1630011

25 (2016) 1643005

25 (2016) 1650008

25 (2016) 1650073

25 (2016) 1641014

25 (2016) 1641021

25 (2016) 1650046

25 (2016) 1650093

25 (2016) 1650108

25 (2016) 1650077

25 (2016) 1650091

25 (2016) 1650097

25 (2016) 1630019

25 (2016) 1650055

25 (2016) 1650076

25 (2016) 1650042

25 (2016) 1630016

25 (2016) 1630031 Coelho

25 (2016) 1641010

Sasaki, M., see Chen

Sasaki, M., see G. Domènech

25 (2016) 1645003

25 (2016) 1645006

Satin, S., Malafarina, D. \& Joshi, P. S., Genericity aspects of black hole formation in the collapse of spherically symmetric slightly inhomogeneous perfect fluids

Savelova, E. P., see Kirillov

Schober, G. A. H., see Starke

Sebastiani, L., see Myrzakul

Sefiedgar, A. S., How can rainbow gravity affect gravitational force?

Seidel, P. T. \& Cabral, L. A., Dualities and geometrical invariants for static and spherically symmetric spacetimes

Senikoglu, Y., see Arik

Setare, M. R., see Darabi

Setare, M. R., see Sahraee

Shankaranarayanan, S., see Skákala

Sharif, M. \& Fatima, H. I., Built-in inflation in $f(G)$ gravity

Sharif, M. \& Fatima, H. I., Static spherically symmetric solutions in $f(G)$ gravity

Sheikh-Jabbari, M. M., Residual diffeomorphisms and symplectic soft hairs: The need to refine strict statement of equivalence principle

Shengelia, T., see Gogberashvili

Sheykhi, A. \& Hajkhalili, S., Extended phase space and thermodynamic geometry of topological Born-Infeld-dilaton black holes

Sheykhi, A., Dehghani, M. H. \& Ghaffari, S., New holographic dark energy model inspired by the DGP braneworld
25 (2016) 1650023

25 (2016) 1650075

25 (2016) 1640010

25 (2016) 1650041

25 (2016) 1650101

25 (2016) 1641007

25 (2016) 1650088

25 (2016) 1650104

25 (2016) 1650097

25 (2016) 1650047

25 (2016) 1650011

25 (2016) 1650083

25 (2016) 1644019

25 (2016) 1630019

25 (2016) 1650062

25 (2016) 1650018 
Shiao, Y.-S., see Nam

Shojaie, H., see Karchi

Shu, F.-W., see Guo

Silagadze, Z. K., see Dirkes

Silberstein, M., see Stuckey

Silva, H. O., Maselli, A., Minamitsuji, M. \& Berti, E., Compact objects in Horndeski gravity

Silva, J. C. J., see Bessa

Singh, K. N., Bhar, P. \& Pant, N., A new solution of embedding class I representing anisotropic fluid sphere in general relativity

Singh, P., Is classical flat Kasner spacetime flat in quantum gravity?

Singh, T. P., see Banerjee

Singleton, D. \& Wilburn, S., Global versus local - Mach's principle versus the equivalence principle

Skákala, J. \& Shankaranarayanan, S., Black hole thermodynamics as seen through a microscopic model of a relativistic Bose gas

Sokołowski, L. M. \& Golda, Z. A., Every timelike geodesic in Anti-de Sitter spacetime is a circle of the same radius

Sokolov, V. A., see Denisov

Soo, C., Quantum geometrodynamics with intrinsic time development

Sotomayor, A., see Bellorín

Sperhake, U., see Berti

Sperhake, U., see Cook

Sporea, C. A. \& Borowiec, A., Low energy greybody factors for fermions emitted by a Schwarzschild-de Sitter black hole

Stahl, C., Inhomogeneous matter distribution and supernovae

Starke, R. \& Schober, G. A. H., Relativistic covariance of Ohm's law

Sten, A. K., see Stuckey
25 (2016) 1645013

25 (2016) 1650045

25 (2016) 1650021

25 (2016) 1650015

25 (2016) 1644004

25 (2016) 1641006

25 (2016) 1641017

25 (2016) 1650099

25 (2016) 1642001

25 (2016) 1644005

25 (2016) 1644009

25 (2016) 1650047

25 (2016) 1650007

25 (2016) 1640003

25 (2016) 1645008

25 (2016) 1650016

25 (2016) 1641022

25 (2016) 1641013

25 (2016) 1650043

25 (2016) 1650066

25 (2016) 1640010

25 (2016) 1644004
Stoica, O. C., Kaluza theory with zero-length extra dimensions

Stuckey, W. M., McDevitt, T., Sten, A. K. \& Silberstein, M., End of a dark age?

Sun, G. \& Huang, Y.C., The cosmology in $f(R, \tau)$ gravity without dark energy

Sun, J.-R., see Guo

Taser, D. \& Dog̃ru, M. U., Conformal and traversable wormholes with monopole and perfect fluid in $f(R)$ gravity

Terada, Y., see Maeda

Timofeev, L. V., see Ivanov

Titarchuk, L., see Frontera

Tossa, J., see Boko

Trombetti, T., see Burigana

Tsamparlis, M., see Paliathanasis

Tsuruta, S., Temperature of neutron stars

Tunyasuvunakool, S., see Cook

Vachaspati, T., Gravitational waves, gamma ray bursts, and black stars

Vakili, B. \& Gorji, M. A., Nonsingular universe from generalized thermostatistics

Valentini, A., see Colin

Varieschi, G. U. \& Ault, K. L., Wormhole geometries in fourth-order conformal Weyl gravity

Viaggiu, S., Entropy, energy and temperature-length inequality for Friedmann universes

Vielva, P., see Burigana

Visser, M., see Boonserm

Visser, M., see Nawarajan

Vukašinac, T., see Corichi

Wang, J. \& Huang, C.-G., BF theory explanation of the entropy for rotating isolated horizons

Wang, M., Boundary conditions for Maxwell
25 (2016) 1640004

25 (2016) 1644004

25 (2016) 1650038

25 (2016) 1650085

25 (2016) 1650017

25 (2016) 1630024

25 (2016) 1650090

25 (2016) 1630014

25 (2016) 1650098

25 (2016) 1630016

25 (2016) 1675001

25 (2016) 1630026

25 (2016) 1641013

25 (2016) 1644025

25 (2016) 1650028

25 (2016) 1650068

25 (2016) 1650064

25 (2016) 1650033

25 (2016) 1630016

25 (2016) 1650019

25 (2016) 1650106

25 (2016) 1630011

25 (2016) 1650100 
fields in Kerr-AdS spacetimes

Wang, M.-T., see Chen

Wang, M.-Z., see Nam

Wang, N., see Li

Wang, P., see $\mathrm{Li}$

Wang, S.-H., see Nam

Wibig, T. \& Wolfendale, A. W., Cosmic ray contributions to the WMAP polarization data on the cosmic microwave background

Wilburn, S., see Singleton

Wilson-Ewing, E., Separate universes in loop quantum cosmology: Framework and applications

Wiltshire, D. L., see Buchert Witten, L., see Contopoulos

Wolfendale, A. W., see Wibig

Wong, H. T., Yue, Q. \& Kang, K., Direct dark matter searches and the CDEX research program

Xu, P., see Qiang

$\mathrm{Xu}, \mathrm{Y}$., see Huang

$\mathrm{Xu}$, Y., see $\mathrm{Li}$

Yang, H. S., Emergent spacetime for quantum gravity

Yang, L. \& Li, Z., Shadow of a dressed black hole and
25 (2016) 1641011

25 (2016) 1645001

25 (2016) 1645013

25 (2016) 1650002

25 (2016) 1650002

25 (2016) 1645013

25 (2016) 1650029

25 (2016) 1644009

25 (2016) 1642002

25 (2016) 1630007

25 (2016) 1650022

25 (2016) 1650029

25 (2016) 1645012

25 (2016) 1650070

25 (2016) 1650059

25 (2016) 1650002

25 (2016) 1645010 determination of spin and

viewing angle

Yang, R.-Q., see Cai

Yau, S.-T., see Chen

Yeom, D.-H., see Chen

Yokoyama, J., Birth of the inflationary universe and tensor fluctuations

Yu, Z., see Guo

Yuan, Q., see Zhao

Yue, Q., see Wong

Yugindro Singh, K., see Ibungochouba Singh

Zanelli, J., see Ayón-Beato

Zaslavskii, O. B., High energy particle collisions and geometry of horizon

Zavala, I., see Parameswaran

Zhao, Y., Yuan, Q., Bi, X.J., Zhu, F.-R. \& Jia, H.-Y., Perspective of detecting very high energy gammaray emission from active galactic nuclei with Large High Altitude Air Shower Observatory (LHAASO)

Zhu, F.-R., see Zhao

Zubair, M., Phantom crossing with collisional matter in $f(T)$ gravity
25 (2016) 1650026

25 (2016) 1645011

25 (2016) 1645001

25 (2016) 1645003

25 (2016) 1645009

25 (2016) 1650021

25 (2016) 1650006

25 (2016) 1645012

25 (2016) 1650061

25 (2016) 1641009

25 (2016) 1650095

25 (2016) 1644011

25 (2016) 1650006

25 (2016) 1650006

25 (2016) 1650057 